Pecvnia, 3 (2006), pp. 189-204

\title{
El autoempleo en los ciudadanos de la Unión Europea ampliada: heterogeneidad de los riesgos percibidos
}

\author{
Luis Miguel Zapico Aldeano \\ Sergio del Cano Rojo
}

La Unión Europea considera que la mejor manera de potenciar el crecimiento social y económico es fomentar las iniciativas emprendedoras pero, desafortunadamente, todavía hay muy pocos europeos estableciendo sus propios negocios. Por ello, el propósito de este estudio es relacionar el interés de los ciudadanos europeos en autoemplearse con los riesgos y barreras que perciben a la hora de enfrentarse a este proceso emprendedor. El análisis planteado cubre la totalidad de la Unión Europea de manera exploratoria, debido fundamentalmente a la falta de datos pasados de los 10 países nuevos miembros de la Unión. Los resultados muestran las grandes diferencias existentes entre los estados miembros y la necesidad de profundizar en la explicación de las dimensiones obtenidas. Desde un punto de vista práctico, se plantea que políticas de promoción de la actividad emprendedora se han de desarrollar fundamentalmente en los ámbitos estatal y regional.

Palabras clave: Actividad emprendedora, riesgos y barreras a la creación de empresas.
The European Union considers that the best way to boost the social and economic growth is to encourage entrepreneurial initiatives but, unfortunately, there are still very few Europeans setting up their own business. Therefore, the aim of this study is to relate the interest of Europeans citizens on being self-employed with the perceived risks and barriers to be faced up in this entrepreneurial process. The analysis covers the entire European Union, working on an exploratory base mainly due to the lack of past data about the ten new European Union Members States. The results show the great differences between the Member States and ask for a deep insight explaining the obtained dimensions. From a practical point of view, it is proposed that entrepreneurship promotion policies should be performed at the national and regional level.

Key words: Entrepreneurial activity, entrepreneurial risks and barriers. 


\section{INTRODUCCIÓN}

La actividad emprendedora supone importantes beneficios, tanto económicos como sociales; pues no constituye únicamente una fuente de creación de empleo ${ }^{1}$, competitividad y crecimiento económico ${ }^{2}$, sino que también contribuye al alcance de objetivos sociales y al desarrollo del individuo ${ }^{3}$. De esta manera, la prosperidad de los pueblos está unida al espíritu de empresa y a la vitalidad emprendedora (Cuervo 2003), por lo que, ante esta situación, las Administraciones públicas vienen desarrollando múltiples medidas para la promoción de dicha actividad emprendedora (Reynolds et al. 2002; Chrisman y McMullan 2002; White y Reynolds 1996). En la actualidad y teniendo en cuenta lo anterior, la actividad emprendedora supone el descubrimiento, evaluación y explotación de oportunidades para introducir nuevos productos y servicios, formas de organización, mercados, procesos y materiales, a partir de esfuerzos organizativos inexistentes previamente (Shane 2003; Shane y Venkataraman 2000; Venkataraman 1997).

La Unión Europea, como organismo supranacional, no es ajena a este fenómeno y a sus implicaciones socioeconómicas y, por ello, desde el Consejo Europeo de Lisboa celebrado marzo de 2000, ha buscado la manera de potenciar la creación de empresas; puesto que se considera una de las iniciativas primordiales para mejorar el empleo, las reformas económicas y la cohesión social ${ }^{4}$. Para esto, la Comisión Europea ha creado en su seno una Dirección General de Empresas; la cuál ha fijado cinco áreas estratégicas: revalorizar la figura del emprendedor, animar a

Según los datos de la Comisión Europea (2004), un 31\% de los europeos -U.E. (25)-, actualmente no empleados por cuenta propia, probablemente recurran al autoempleo en los cinco próximos años. Otro ejemplo lo proporciona el panel Global Entrepreneurship Monitor, con datos relativos a 34 países, el cuál estimó que en el año 2004 el $9 \%$ de las personas en edad de trabajar estaban implicadas en la creación de nuevos negocios (Instituto de Empresa 2005).

2 Véase, entre otros, a Reynolds et al. (2005), Baumol (2004, 1993), Brown y Uljin (2004), Shane y Venkataraman (2003, 2000), Genescá et al. (2003), Audretsch y Thurik (2001), McGrath (1999), Wennekers y Thurik (1999), Hatten (1997), Casson (1992), Hornaday (1992), Low y McMillan (1988).

3 Según los datos de la Comisión Europea (2004), los ciudadanos europeos -U. E. (25) - que preferirían estar en una situación de autoempleo justificarían esta decisión, principalmente, por la independencia o autosatisfacción que asocian a este estatus y al interés de las tareas que conlleva $(77 \%)$, seguido a mucha distancia por la consecución de mejores ingresos $(23 \%$ y por otras razones diversas -oportunidad de negocio, coyuntura económica, etcétera-.

$4 \quad$ En la línea de lo expuesto en dicho Consejo surge el documento "Construir una Europa Empresarial. Actividades de la Unión a favor de la Pyme", de la Comisión de las Comunidades Europeas, con el objetivo de sentar las bases para el establecimiento de un clima favorable a la creación y desarrollo de empresas, y en particular de Pymes. 
los ciudadanos a convertirse en empresarios, ayudar al empresario a crecer y ser más competitivo, mejorar los flujos financieros y crear un marco político-legal más adecuado para las PYMEs.

A pesar de estos esfuerzos, según los datos de la propia Comisión Europea (2004), la actividad emprendedora en Europa es escasa, especialmente si la comparación se realiza con EE.UU.:

- Los europeos prefieren estar empleados por cuenta ajena (50\%) mientras que los estadounidenses prefieren estar autoempleados $(61 \%)$.

- Los europeos no se plantean crear negocios (64\%) mientras que los estadounidenses sí (sólo el 44\% no lo considera).

- En un futuro a medio plazo, los europeos no perciben el autoempleo como una opción ni deseable (63\%), ni factible (67\%), mientras que los estadounidenses no lo consideran ni tan indeseable (50\%), ni tan imposible (54\%).

- Los europeos no están inmersos en actividades emprendedoras (88\%), frente a la mayor actividad de los estadounidenses (74\% no realizan estas actividades).

Por ello, aún permanecen vigentes las dos cuestiones centrales planteadas por la Comisión Europea en el Documento Verde "Creación de Empresas en Europa" (2003):

- ¿Por qué hay tan pocos europeos creando sus propios negocios?.

- ¿Por qué crecen tan pocos negocios en Europa?.

Este trabajo trata de buscar respuestas a la primera de las dos cuestiones, la escasez de interés de los ciudadanos europeos en las actividades emprendedoras, mediante los siguientes objetivos:

1. Identificar las principales razones del interés de los ciudadanos de la Unión Europea por la actividad emprendedora.

2. Establecer comparaciones entre los distintos países miembros de la Unión. 
La falta de datos sobre las percepciones de los ciudadanos, especialmente de los 10 países recién incorporados a la Unión, ha obligado a la realización de un análisis exploratorio. No obstante, teniendo en cuenta esta limitación, la metodología utilizada permite presentar unas dimensiones subyacentes al fenómeno de manera comparada entre los países de la UE; dimensiones pueden servir de punto de partida para profundizar en posteriores trabajos.

\section{MARCO TEÓRICO}

La falta de consenso en una teoría general de la creación de empresas procede de la amplitud del campo de investigación, tanto por la diversidad de factores que influyen como por la existencia de distintos niveles de análisis. Su consecuencia ha sido la proliferación de estudios parciales, tanto en variables como en casos, y la creciente necesidad de buscar teorías de carácter general e integrador y estudios empíricos que aporten una visión global del proceso (Fernández y Junquera 2001). Con este trabajo se inicia el proceso de realización de uno de esos estudios empíricos que puedan aportar una visión más globalizada del proceso para el caso de la Unión Europea.

Teniendo en cuenta lo anteriormente citado, es preciso indicar previamente la aproximación teórica que sustenta el estudio. Este marco general es la consideración de la creación de empresas como el fenómeno, a lo largo del tiempo, de la emergencia de oportunidades económicas y su explotación en nuevas empresas y/o industrias, incorporando tanto los efectos individuales como los efectos de las oportunidades y de los entornos industrial e institucional. Concretamente, la economía opera en un estado continuo de desequilibrio, los cambios -tecnológicos, políticos, sociales- ocurren y generan situaciones en las que las personas pueden transformar recursos en algo nuevo - productos y servicios, procesos productivos, formas de organización, materiales, mercados- que tiene más valor que el coste de su creación. En consecuencia, la creación de empresas es un fenómeno multidisciplinar y complejo que no se desenvuelve únicamente en un contexto organizativo o sociológico, ni sólo consiste en una tipología de conductas, ni únicamente es un modelo de desarrollo político-económico (Venkataraman 1997; Shane y Venkataraman 2000; Shane 2003).

Dentro de este complejo fenómeno, el descubrimiento de oportunidades de negocio supone que el individuo ha de crearse conjeturas 
a partir de información específica o de creencias, de manera que vea oportunidades donde otros ven riesgos. Por otra parte, la decisión de explotar esas oportunidades de negocio detectadas supone la valoración conjunta de tales conjeturas y el convencimiento de estar en posesión de la capacidad necesaria para la explotación adecuada y ventajosa de dicha oportunidad (Ardichvili et al. 2003). En esta primera aproximación agruparemos dichas valoraciones en los siguientes aspectos: actitudes hacia el autoempleo, riesgos y dificultades del proceso.

\section{METODOLOGÍA}

\subsection{Datos}

Los datos empleados en el estudio proceden del Eurobarómetro sobre Creación de Empresas, elaborado por la Comisión Europea. A pesar de la existencia de otros estudios especializados sobre el tema, por ejemplo los elaborados por la OCDE o el proyecto Global Entrepreneurship Monitor, el Eurobarómetro se ha escogido por su idoneidad para alcanzar los objetivos de este trabajo: su mayor cobertura específica poblacional supone que el grado de comparabilidad entre los resultados obtenidos para los diferentes países sea considerablemente superior al de cualquier otro conjunto de datos sobre la creación de empresas en los Estados Miembros de la UE.

TABLA 1. Tamaño muestral

\begin{tabular}{|l|r|r|}
\hline \multicolumn{1}{|c|}{ País } & Muestra & \multicolumn{1}{c|}{$\%$} \\
\hline Bélgica & 419 & 2,3 \\
\hline Dinamarca & 216 & 1,2 \\
\hline Alemania & 3.428 & 18,5 \\
\hline Grecia & 443 & 2,4 \\
\hline España & 1.659 & 8,9 \\
\hline Francia & 2.390 & 12,9 \\
\hline Irlanda & 148 & 0,8 \\
\hline Italia & 2.450 & 13,2 \\
\hline Luxemburgo & 18 & 0,1 \\
\hline Países Bajos & 644 & 3,5 \\
\hline Austria & 344 & 1,8 \\
\hline Portugal & 413 & 2,2 \\
\hline Finlandia & 210 & 1,1 \\
\hline Suecia & 358 & 1,9 \\
\hline Reino Unido & 2.396 & 12,9 \\
\hline Chipre & 29 & 0,2 \\
\hline República Checa & 424 & 2,3 \\
\hline Estonia & 55 & 0,3 \\
\hline
\end{tabular}




\begin{tabular}{|l|r|r|}
\hline \multicolumn{1}{|c|}{ País } & Muestra & \multicolumn{1}{c|}{$\%$} \\
\hline Hungría & 412 & 2,2 \\
\hline Letonia & 96 & 0,5 \\
\hline Lituania & 147 & 0,8 \\
\hline Malta & 16 & 0,1 \\
\hline Polonia & 1.545 & 8,3 \\
\hline Eslovaquia & 214 & 1,2 \\
\hline Eslovenia & 83 & 0,4 \\
\hline UE (25) & 18.547 & 100,0 \\
\hline
\end{tabular}

A pesar de la existencia de datos del Eurobarómetro desde el año 2000, se han tenido que analizar sólo los correspondientes al año 2004 porque, obviamente, es la primera oleada que incluye información sobre los nuevos países incorporados a la Unión Europea. No obstante, la fiabilidad de los resultados está asegurada dado el tamaño de la muestra y los métodos de muestreo utilizados (Comisión Europea 2004).

La muestra contiene los datos de un total de 18.547 ciudadanos de la Unión Europea correspondiendo a cada país un número de entrevistas ponderado proporcionalmente a la participación del mismo en la UE (Tabla 1).

\subsection{Variables}

El interés de los ciudadanos europeos por la actividad emprendedora se ha recogido, inicialmente, a través de una escala nominal que refleja la preferencia por el autoempleo o por ser empleado. A partir de la distribución de esta variable se ha creado una variable nueva "Interés emprendedor (IE)", medida en una escala ordinal de tres niveles (alto, medio, bajo), que es la que se ha utilizado en este análisis comparado.

Los riesgos asociados a la actividad emprendedora se han calculado, en un principio, a partir de una escala nominal abierta que muestra los riesgos más temidos por los ciudadanos a la hora de establecer un negocio (la incertidumbre en los ingresos, la inseguridad laboral, la pérdida patrimonial, el esfuerzo excesivo, el fracaso personal y la bancarrota). Para obtener una única escala se ha promediado cada nivel obtenido en la escala inicial de forma ponderada según la frecuencia mostrada. Análogamente al caso anterior, tras comprobar la fiabilidad de la esta única escala $(\alpha=0,86)$, a partir de la distribución de la misma se ha se creado la variable "Nivel de riesgo (NR)", medida en una escala ordinal de tres niveles (excesivo, normal, escaso). 
Las barreras a la creación de nuevos negocios se han estimado a partir de 5 variables, medidas en escalas Likert de 5 puntos, que reflejan el grado de acuerdo de los ciudadanos con las principales barreras mostradas tradicionalmente por la literatura: la falta de apoyos financieros, la complejidad administrativa, el contexto económico, la posibilidad de fracasar y la falta de información. Para conseguir una escala única se ha promediado cada nivel de esas variables, y cada variable entre sí, de forma ponderada según las frecuencias mostradas. Tras comprobar la fiabilidad de la escala resultante $(a=0,83)$, de la misma manera que en el caso precedente, a partir de su distribución, se ha creado la variable "Barreras (BA)", medida en una escala ordinal de tres niveles (muchas, regulares, pocas).

\subsection{Técnica de análisis de datos}

El análisis multidimensional es el más apropiado como técnica exploratoria para identificar dimensiones no reconocidas que afectan al comportamiento y como medio de obtener evaluaciones comparativas de objetos cuando las bases de comparación no están definidas (Hair et al. 1999). La técnica es más útil cuando un extenso número de variables impide una interpretación eficaz de las relaciones entre los objetos (sujetos y unidades); así, al reducir la dimensionalidad, se interpreta un pequeño número de componentes en lugar de un extenso número de variables.

Consecuentemente, la técnica de análisis de datos que más se ajusta a nuestros objetivos de caracterizar y comparar a los ciudadanos de los 26 países miembros de la Unión Europea, según la percepción que presentan respecto a la actividad emprendedora, es el análisis de correspondencias múltiples. Esta técnica de interdependencia de datos facilitará, por un lado, la reducción dimensional de una clasificación de objetos - ciudadanos de los estados de la UE- sobre un conjunto de atributos - percepciones sobre los riesgos y barreras asociados a la actividad emprendedora- $y$, por otro lado, permitirá la representación perceptual subjetiva y conjunta de los objetos relativos a tales atributos. Adicionalmente al énfasis que pone en la percepción de los individuos, esta herramienta se escoge sobre otras técnicas de interdependencia de datos, por ejemplo el análisis de conglomerados o el análisis discriminante, 
por su capacidad para emplear tanto datos métricos como relaciones no lineales ${ }^{5}$.

\section{RESULTADOS DEL ANÁLISIS EMPÍRICO Y DISCUSIÓN}

En la presentación de los resultados vamos a distinguir entre los dos tipos de análisis realizados: el estudio de los factores que influyen sobre el interés por la actividad emprendedora y la comparación conjunta para los distintos países de la Unión.

\subsection{Determinantes externos del comportamiento}

emprendedor

A continuación se indica el resumen de datos para todas las variables iniciales, agrupadas según las variables finales y por países (Tabla 2).

TABLA 2. Percepciones de los ciudadanos de la UE(25) sobre la actividad emprendedora

\begin{tabular}{|c|c|c|c|c|c|c|c|c|c|c|c|c|c|}
\hline \multirow{2}{*}{\multicolumn{2}{|c|}{ PAÍS }} & INTERÉS & \multicolumn{6}{|c|}{ RIESGOS } & \multicolumn{5}{|c|}{ BARRERAS } \\
\hline & & 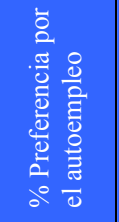 & 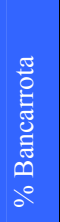 & 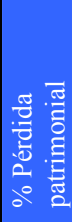 & 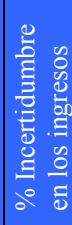 & 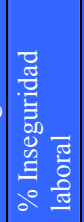 & 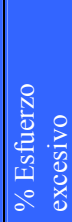 & 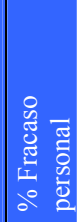 & 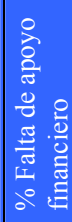 & 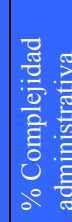 & 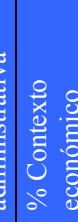 & 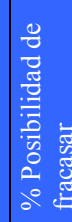 & 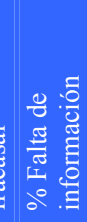 \\
\hline Bélgica & $\mathrm{BE}$ & 34 & 48 & 25 & 39 & 18 & 16 & 12 & 74 & 71 & 64 & 52 & 60 \\
\hline Dinamarca & DK & 38 & 42 & 31 & 34 & 11 & 29 & 11 & 53 & & 44 & 51 & 34 \\
\hline Alemania & DE & 39 & 47 & 50 & 30 & 17 & 12 & 15 & 72 & 72 & 74 & 61 & 43 \\
\hline Grecia & EL & 52 & 42 & 17 & 48 & 38 & 11 & 25 & 89 & 74 & 81 & 43 & 61 \\
\hline España & ES & 56 & 41 & 35 & 23 & 16 & 10 & 15 & 73 & 67 & 55 & 44 & 47 \\
\hline Francia & FR & 42 & 47 & 29 & 25 & 19 & 16 & 19 & 80 & 72 & 67 & 43 & 18 \\
\hline Irlanda & IE & 58 & 45 & 31 & 28 & 13 & 22 & 15 & 60 & 64 & 36 & 29 & 33 \\
\hline Italia & IT & 55 & 44 & 17 & 43 & 18 & 17 & 13 & 87 & 77 & 82 & 51 & 57 \\
\hline Luxemburgo & LU & 48 & 55 & 37 & 29 & 15 & 12 & 19 & 78 & 64 & 62 & 55 & 45 \\
\hline Países Bajos & NL" & 33 & 38 & 25 & 44 & 22 & 21 & 10 & 45 & 58 & 63 & 44 & 19 \\
\hline Austria & AT & 37 & 42 & 34 & 29 & 15 & 18 & 14 & 66 & 56 & 57 & 61 & 30 \\
\hline Portugal & PT & 62 & 48 & 34 & 30 & 14 & 8 & 15 & 82 & 78 & 82 & 62 & 66 \\
\hline Finlandia & FI & 28 & 28 & 30 & 40 & 9 & 32 & 10 & 43 & 60 & 43 & 41 & 21 \\
\hline
\end{tabular}

5 Este método de escalamiento óptimo permite escalar las variables a diferentes niveles, cuantificando las variables categóricas de forma óptima en la dimensionalidad especificada. Como resultado, se pueden modelar relaciones no lineales entre las variables. 


\begin{tabular}{|c|c|c|c|c|c|c|c|c|c|c|c|c|c|}
\hline \multirow[b]{2}{*}{ PAÍS } & & \multirow{2}{*}{ 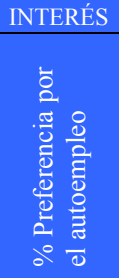 } & \multicolumn{6}{|c|}{ RIESGOS } & \multicolumn{5}{|c|}{ BARRERAS } \\
\hline & & & 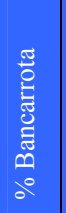 & 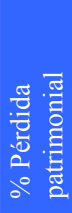 & 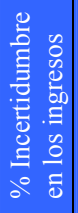 & 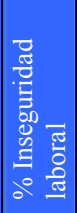 & 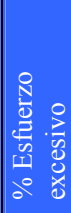 & 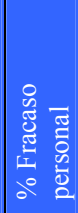 & 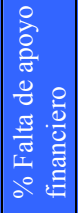 & 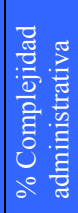 & $\begin{array}{ll}0 & 0 \\
0 & 0 \\
0 & 0 \\
0 & 0 \\
0 & 0 \\
0 & 0 \\
0 & 0\end{array}$ & 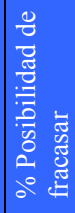 & 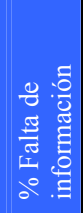 \\
\hline Suecia & $\mathrm{SE}$ & 35 & 35 & 28 & 28 & 16 & 30 & 7 & 65 & 68 & 61 & 49 & 38 \\
\hline Reino Unido & & 41 & 47 & 47 & 38 & 15 & 18 & 16 & 57 & 61 & 42 & 43 & 34 \\
\hline Chipre & & 56 & 26 & 27 & 31 & 17 & 21 & 17 & 80 & 61 & 65 & 53 & 49 \\
\hline República Checa & $\mathrm{CZ}$ & 30 & 52 & 34 & 41 & 29 & 17 & 10 & 74 & 70 & 77 & 61 & 40 \\
\hline Esto & E & 37 & 43 & 25 & 24 & 19 & 13 & 16 & 59 & 55 & 49 & 58 & 28 \\
\hline Hun & $\mathrm{HU}$ & 42 & 37 & 48 & 50 & 19 & 14 & 13 & 83 & 74 & 61 & 80 & 48 \\
\hline Letonia & LV & 42 & 54 & 36 & 35 & 17 & 10 & 17 & 93 & 74 & 65 & 62 & 34 \\
\hline Lituani & LT & 52 & 65 & 52 & 19 & 18 & 11 & 20 & 81 & 83 & 70 & 69 & 50 \\
\hline Malta & MT & 44 & 43 & 13 & 34 & 21 & 20 & 40 & 78 & 57 & 65 & 70 & 27 \\
\hline Polonia & PL & 51 & 52 & 31 & 35 & 9 & 11 & 14 & 87 & 70 & 70 & 56 & 48 \\
\hline Eslovaquia & SK & 30 & 48 & 35 & 31 & 28 & 14 & 9 & 93 & 77 & 76 & 60 & 51 \\
\hline Eslovenia & SI & 32 & 53 & 36 & 31 & 15 & 11 & 22 & 84 & 79 & 69 & 69 & 48 \\
\hline JNIÓN EUROPE $f$ & UE & 45 & 45 & 35 & 34 & 17 & 15 & 5 & 74 & 70 & 66 & 51 & 45 \\
\hline
\end{tabular}

Los resultados globales presentan grandes variaciones entre los países miembros de la Unión, no detectándose a priori explicaciones de afinidad geográfica o del momento de integración.

El interés por la actividad emprendedora oscila desde el $28 \%$ de finlandeses interesados en el autoempleo hasta el 62\% de portugueses. Francia, Reino Unido, Letonia, Malta, Luxemburgo y Hungría presentan tasas de preferencia similares a la media de la Unión. Destacan los casos de Portugal, y en menor medida de Irlanda, España, Chipre e Italia como estados con mayores porcentajes de aceptación del autoempleo.

Los riesgos más relevantes para los ciudadanos europeos son la bancarrota, la pérdida patrimonial y la incertidumbre en los ingresos de la actividad. Importantes, en menor medida, son la inseguridad laboral, la necesidad de realizar grandes esfuerzos y el temor al fallo personal:

- Respecto a la posibilidad de bancarrota tenemos que los ciudadanos de Irlanda, Italia, Francia, Reino Unido, Estonia y Malta presentan percepciones similares al promedio de la Unión. Destaca el temor de los lituanos (65\%) que contrasta con la escasa puntuación de los chipriotas (26\%). Esta variable, junto a la posterior, son las que presentan mayor rango de variación. 
- La pérdida patrimonial es un riesgo que preocupa especialmente a los lituanos (52\%), alemanes, húngaros y británicos. En contraposición, los malteses sólo temen este riesgo en un 13\% de los encuestados.

- Destacan Hungría (50\%) y Grecia como estados donde la incertidumbre de los ingresos de los autoempleados tiene mayor incidencia. Por el contrario, es un riesgo poco importante en España y en Lituania (19\%).

- La mayoría de los países se sitúan en torno al promedio de la Unión para el caso de la inseguridad laboral. Únicamente se ha de mencionar los casos de Polonia y Finlandia (9\%) caracterizados por la escasa incidencia de esta variable, contrastando con la importancia que asume en Grecia (38\%).

- La percepción que la actividad emprendedora precisa de excesivos esfuerzos es un riesgo que preocupa muy poco a los portugueses (8\%), mientras que es un factor mucho más importante para los países escandinavos (Finlandia, Suecia y Dinamarca).

- El temor al fallo personal es una variable que, análogamente a la inseguridad laboral, aglutina a la mayor parte de los estados alrededor de la media de la Unión. No obstante, aparecen casos extremos como el de Suecia $(7 \%)$ y Malta $(40 \%)$.

La importancia de las barreras percibidas por los ciudadanos europeos depende, fundamentalmente, de la falta de apoyo financiero, las trabas burocráticas y el contexto económico; mientras que, en un segundo nivel, influyen la posibilidad de fracasar y la falta de información:

- La falta de apoyo financiero es una barrera muy importante para eslovacos y lituanos (93\%) que contrasta con la escasa puntuación para finlandeses (43\%). Esta variable, junto a la posibilidad de fracasar, son las que presentan mayor rango de variación.

- La complejidad administrativa es la variable con menos rango de variación oscilando entre los casos de Lituania (83\%) y Estonia (55\%).

- Destacan Italia y Portugal (82\%) como estados donde el contexto económico tiene mayor incidencia. Por el contrario, es una barrera poco importante en Irlanda (36\%). 
- Respecto a la posibilidad de fracasar se ha de mencionar el caso de Irlanda (29\%), caracterizada por la escasa incidencia de esta variable; lo que contrasta con la importancia que asume en Hungría (80\%).

- La falta de información para crear un nuevo negocio es una barrera que preocupa muy poco a los neerlandeses (19\%), mientras que es un factor mucho más importante para portugueses (66\%).

\subsection{Análisis comparado}

El siguiente paso en la investigación es la representación de la correspondencia de las categorías de las variables anteriores, proceso que se ha llevado a cabo mediante el programa HOMALS (Análisis de Componentes Principales Categóricos) del paquete estadístico SPSS. Este procedimiento de composición cuantifica simultáneamente las variables categóricas a la vez que reduce la dimensionalidad de los datos con el objetivo de reducir el conjunto original de variables en un conjunto más pequeño de componentes no correlacionados, de manera que representen la mayor parte de la información encontrada en las variables originales.

La optimización de las escalas de las variables iniciales para la aplicación de esta técnica, ha llevado al cálculo de nuevas variables categóricas ordinales (Tabla 3).

TABLA 3. Niveles de las variables del escalamiento óptimo multidimensional

\begin{tabular}{|c|c|c|c|c|}
\hline \multicolumn{2}{|l|}{ PAÍS } & $\begin{array}{c}\text { INTERÉS } \\
\text { EMPRENDEDOR (IE }\end{array}$ & NIVEL DE RIESGO $(N R)$ & BARRERAS $(B A)$ \\
\hline Bélgica & $\mathrm{BE}$ & BAJO & NORMAL & REGULARES \\
\hline Dinamarca & DK & MEDIO & NORMAL & POCAS \\
\hline Alemania & DE & BAJO & EXCESIVO & REGULARES \\
\hline Grecia & EL & ALT & EXCESIVO & MUCHAS \\
\hline España & ES & AL7 & ESCASO & REGULARES \\
\hline Francia & FR & MEDIO & NORMAL & REGULARES \\
\hline Irlanda & IE & ALT & NORMAL & POCAS \\
\hline Italia & IT & AL7 & NORMAL & MUCHAS \\
\hline Luxemburgo & LU & AL & EXCESIVO & REGULARES \\
\hline Países Bajos & NL & BAJO & NORMAL & POCAS \\
\hline Austria & AT & BAJO & NORMAL & POCAS \\
\hline Portugal & PT & $\mathrm{ALT}$ & NORMAL & MUCHAS \\
\hline Finlandia & FI & BAJO & ESCASO & POCAS \\
\hline Suecia & SE & BAJO & ESCASO & POCAS \\
\hline Reino Unido & UK & MEDIO & EXCESIVO & POCAS \\
\hline Chipre & CY & $\mathrm{AL} 7$ & ESCASO & REGULARES \\
\hline República Checa & $\mathrm{CZ}$ & BAJO & EXCESIVO & REGULARES \\
\hline
\end{tabular}




\begin{tabular}{|c|c|c|c|c|}
\hline \multicolumn{2}{|c|}{ PAÍS } & $\begin{array}{c}\text { INTERÉS } \\
\text { EMPRENDEDOR }(I E)\end{array}$ & NIVEL DE RIESGO $(N R) \mid$ & BARRERAS $(B A)$ \\
\hline Estonia & EE & MEDIO & ESCASO & POCAS \\
\hline Hungría & $\mathrm{HU}$ & MEDIO & EXCESIVO & MUCHAS \\
\hline Letonia & LV & MEDIO & EXCESIVO & MUCHAS \\
\hline Lituania & LT & ALTO & EXCESIVO & MUCHAS \\
\hline Malta & MT & MEDIO & NORMAL & REGULARES \\
\hline Polonia & PL & ALTO & NORMAL & MUCHAS \\
\hline Eslovaquia & SK & BAJO & EXCESIVO & MUCHAS \\
\hline Eslovenia & SI & BAJO & EXCESIVO & MUCHAS \\
\hline
\end{tabular}

Para evaluar el ajuste conjunto se han seleccionado el número de dimensiones en función del nivel de variación explicado y de la ganancia de explicación por el añadido de nuevas dimensiones. Usando el criterio de incluir en la solución el número de dimensiones cuya inercia supere el valor 0,2 se han obtenido dos dimensiones, siendo válidos los indicadores del análisis (Tabla 4).

TABLA 4. Dimensiones retenidas en el análisis y sus medidas de discriminación

\begin{tabular}{|l|r|r|r|}
\hline & DIMENSIÓN 1 & DIMENSIÓN 2 & MEDIA \\
\cline { 1 - 2 } & & & \\
\hline Interés emprendedor $(\mathrm{Ie})$ & 0,223 & 0,559 & 0,391 \\
\hline Nivel de riesgo $(\mathrm{Nr})$ & 0,527 & 0,241 & 0,384 \\
\hline Barreras $(\mathrm{Ba})$ & 0,840 & 0,328 & 0,584 \\
\hline País & 1,000 & 1,000 & 1,000 \\
\hline AUTOVALORES & 2,590 & 2,128 & 2,359 \\
\hline INERCIA & 0,648 & 0,532 & 0,590 \\
\hline \% TOTAL DE LA VARIANZA & 64,758 & 53,196 & 58,977 \\
\hline ALFA DE CRONBACH & 0,819 & 0,707 & 0,768 \\
\hline
\end{tabular}

La primera dimensión, eje de abcisas, hace referencia básicamente a las barreras y, en menor medida, al los riesgos asociados a la actividad emprendedora. La dimensión segunda, eje de ordenadas, representa fundamentalmente el interés emprendedor y la existencia de barreras. Las agrupaciones de ciudadanos en países se reparten en el mapa de manera que se sitúan cerca de las categorías de las variables a las que se asocian en mayor medida (Gráfico 1). 
GRÁFICO 1. Posicionamiento según país, interés emprendedor, riesgos y barreras en la creación de negocios

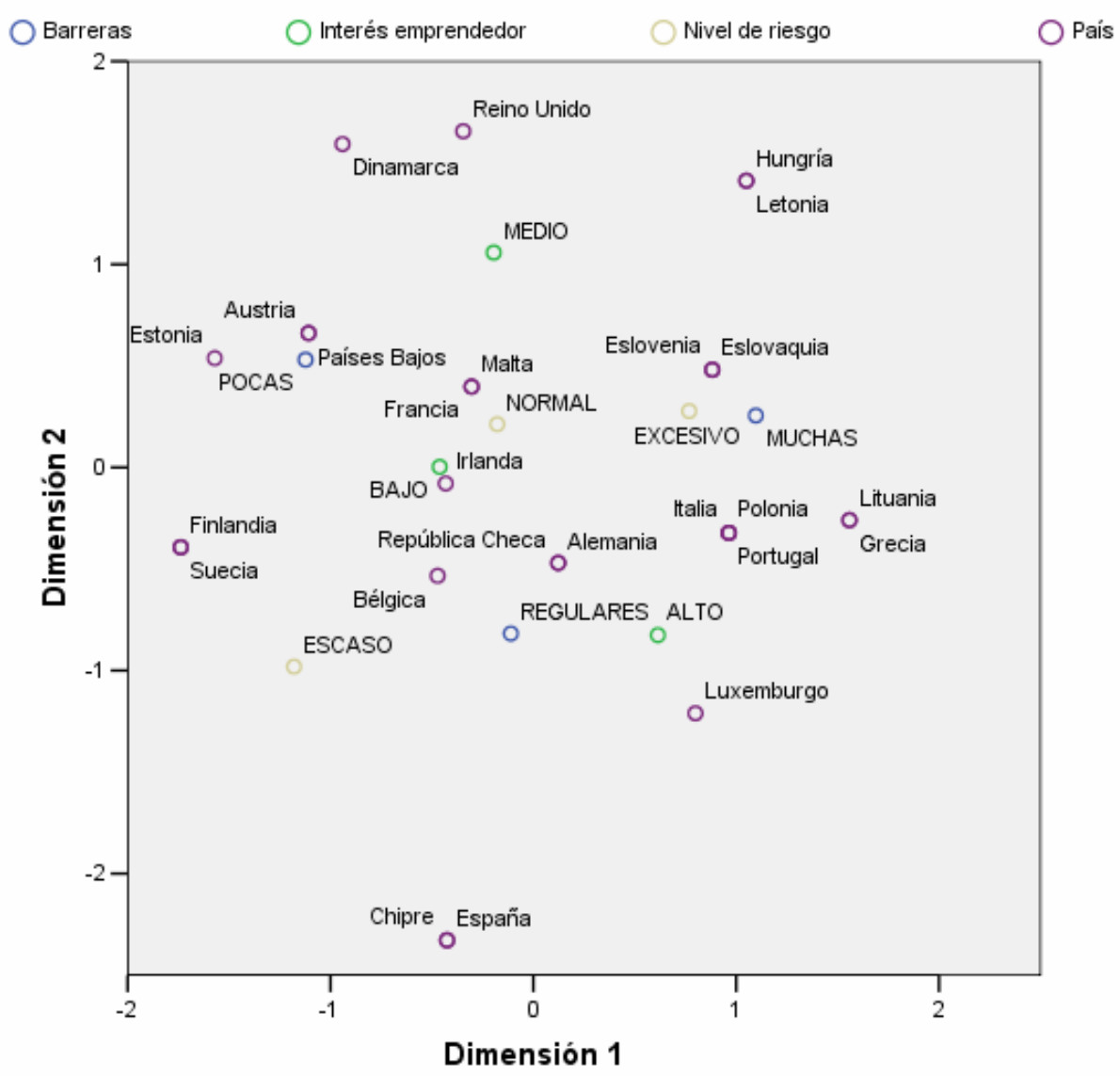

Los resultados obtenidos ponen de manifiesto la existencia de distintos patrones perceptivos según los países. No hay una conducta clara asociada al hecho de ser un país de nuevo ingreso en la Unión ni a otras variables como la renta per cápita o las tasas de crecimiento económico (correlaciones no relevantes) o como la proximidad geográfica (destacando como ejemplo de disparidad la situación de los países de la Península Ibérica o los estados ribereños del mar Báltico).

Atendiendo a las barreras y riesgos asociados a la actividad emprendedora, dimensión 1, la mayor parte de los países de la UE operan en entornos percibidos como moderadamente restrictivos. Los estados de nueva incorporación, en general, consideran que sus entornos son demasiado adversos o cercanos a esta situación; quizá derivado del hecho de las necesarias reformas exigidas por la integración. 
Respecto al interés emprendedor y la existencia de barreras, dimensión 2, la mayor parte de los países de la UE operan en entornos percibidos como moderadamente restrictivos y de escaso interés para la actividad emprendedora. Destacan los casos extremos de Chipre y España, donde se conjugan el alto interés por la actividad emprendedora con la existencia de barreras moderadas.

En definitiva estos resultados corroboran los hallazgos descritos anteriormente en este trabajo.

\section{CONCLUSIONES}

En este trabajo se ha llevado a cabo un análisis comparativo del interés de la activad emprendedora en los países de la Unión Europea así como de los riesgos y barreras a ella asociados. Para ello se han utilizado datos recientes del Eurobarómetro de Creación de Empresas, elaborado por la Comisión Europea, debido a su amplio tamaño muestral. No obstante, el hecho de carecer de datos longitudinales sobre la totalidad de los países de la Unión limita el carácter del estudio hacia un análisis exploratorio. Teniendo en cuenta estas limitaciones, se utilizó como herramienta metodológica el análisis de correspondencias. Este presenta como ventaja la posibilidad de describir conjunta e explícitamente las dimensiones del espacio perceptual y su representación espacial.

Los resultados obtenidos ponen de manifiesto la existencia de distintos patrones perceptivos según los países. No hay una conducta clara asociada al hecho de ser un país de nuevo ingreso en la Unión ni a otras variables como la renta per cápita o las tasas de crecimiento económico o como la proximidad geográfica. Por ello, desde un punto de vista práctico, sería recomendable que las políticas de promoción de la actividad emprendedora se desarrollaren fundamentalmente en los ámbitos estatal y regional.

No obstante, es preciso tener en cuenta los inconvenientes derivados de la composición de atributos: necesidad de incluir todos los atributos destacados, elegir el método de similitud que mejor refleje el pensamiento de los encuestados y la necesidad de tener datos abundantes y fiables sobre los individuos. En la medida en que se vayan obteniendo nuevas oleadas del Eurobarómetro sería oportuna la realización de estudios dinámicos explicativos de los resultados de este trabajo, concretamente de las relaciones de los centroides de las dimensiones obtenidas. 


\section{BIBLIOGRAFÍA}

ARDICHVILI, A.; CARDOZO, R. y SOURAV, R. (2003) "A theory of entrepreneurial opportunity identification and development", Journal of Business Venturing, vol. 18, $\mathrm{n}^{\circ} .1$, pp. 105-123.

AUDRETSCH, D. y THURIK, A. (2001) "What's new about the new economy? Sources of growth in the managed and entrepreneurial economies", Industrial and Corporate Change, vol. 10, $\mathrm{n}^{\circ} 1$, pp. 267-315.

BAUMOL, W.J. (1993) "Formal entrepreneurship theory in economics: existence and bounds", Journal of Business Venturing, vol. 8, $\mathrm{n}^{\circ} 2$, pp. 197-210.

- (2004) "Entrepreneurial cultures and countercultures", Academy of Management Learning and Education, vol. 3, $\mathrm{n}^{\circ} 3$, pp. 316-326.

BROWN, T.E. y ULJIN, J.M. (2004) Innovation, entrepreneurship and culture: the integration between technology, progress and economic growth. Cheltenham (Reino Unido): Edward Elgar Publishers.

CASSON, M. (1992) The entrepreneur. New Jersey (EEUU): Barnes \& Noble Books.

Chrisman, J. y MCMUllan, W. (2002) "Some Additional Comments on the Sources and Measurement of the Benefits of Small Business Assistance Programs", Journal of Small Business Management, vol. 40, $\mathrm{n}^{\circ} 1$, pp. 43-50.

COMISIÓN EUROPEA (2001) "Construir una Europa empresarial. Actividades de la Unión en favor de la pequeña y mediana empresa (PYME)", Documento de la Comisión de las Comunidades Europeas, COM (2001) 98.

- (2003) Green Paper: Entrepreneurship. Bruselas: Comisión Europea.

- (2004) Eurobarometer: Entrepreneurship. Bruselas: Comisión Europea.

CUeRVo, A. (2003) "La creación empresarial: de empresarios y directivos". GenesCÁ, E.; URBANO, D.; CAPELleras, J.L.; Guallarte, C. y Vergés, J. (2003) Creación de Empresas: homenaje al profesor José María Veciana Vergés. UAB, pp. 49-74.

FERNÁNDEZ SÁNCHEZ, E. y JUNQUERA, B. (2001) "Factores determinantes en la creación de pequeñas empresas: una revisión de la literatura", Papeles de Economía Española, $\mathrm{n}^{\circ}$. 89/90, pp. 322-342.

Genescá, E.; URBano, D.; CAPelleras, J.L.; Guallarte, C. y Vergés, J. (2003) Creación de Empresas: homenaje al profesor José María Veciana Vergés. Barcelona: UAB. 
HAIR JR, J.F.; ANDERSON, R.E.; TATHAM, R.L. y BLACK, W.C. (1999) Análisis multivariante ( $5^{\mathrm{a}}$ edición). Madrid: Prentice Hall.

HATTEN, T.S. (1997) Small Business: Entrepreneurship and Beyond. Upper Saddle River, NJ: Prentice Hall.

HORNADAY, R.W. (1992) "Thinking about entrepreneurship: a fuzzy set approach", Journal of Small Business Management, vol. 30, $\mathrm{n}^{\circ} 3$, pp. $12-23$.

INSTITUTO DE EMPRESA (2005) Global entrepreneurship monitor. Informe Ejecutivo (2004) - España. Madrid: IE.

LOW, M.B. y MCMILLAN, I.C. (1988) "Entrepreneurship: past research and future challenges", Journal of Management, vol. 14, n 1, pp. 110-131.

MCGRATH, R.G. (1999) "Falling forward: real options reasoning and entrepreneurial failure", Academy of Management Review, vol. 24, $\mathrm{n}^{\circ}$ 1, pp. 13-30.

ReYNoldS, P.D.; BygraVe, W.D.; Autio, E.; Cox, L.W. y HaY, M. (2002) Global Entrepreneurship Monitor. Executive Report (2002), Wellesley, MA: Babson College.

-; Bosma, N.; Autio, E.; Hunt, S.; De Bono, N.; Servais, I.; LóPez-García, P. y CHIN, N. (2005) "Global Entrepreneurship Monitor: data collection, design and implementation", Small Business Economy, vol. 24, n² 2, pp. 205-231.

Shane, S. y VenKataRaman, S. (2000) "The promise of entrepreneurship as a field of research", Academy of Management Review, vol. 25, $\mathrm{n}^{\circ} 1$, pp. 217-226.

- (2003) A general theory of entrepreneurship: the individual-opportunity nexus. Cheltenham (Reino Unido): Edward Elgar Publishers.

VenKatARAMAN, S. (1997) "The distinctive domain of entrepreneurship research: An editor's perspective". KATZ, J. and BORCKHAUS, R. (eds.) Advances in Entrepreneurship, Firm Emergence and Growth, 3, JAI Press, pp. 119-138.

WeNNEKERS, A. y THURIK, A. (1999) "Linking entrepreneurship and economic growth", Small Business Economics, vol. 13, n 1, pp. 27-55.

WHITE, S.B. y REYNOLDS, P. (1996) "Government programs and high growth new firm", Frontiers of Entrepreneurship Research, Center for Entrepreneurial Studies Babson College. Wellesley, MA. 